\title{
DOUBLE CONDUCTOR LINE ABOVE A TWO-LAYERED CYLINDER WITH VARYING PROPERTIES
}

\author{
A. A. KOLYSHKIN ${ }^{1}$ and RÉMI VAILLANCOURT ${ }^{2}$
}

(Received 4 July 1994; revised 3 September 1994)

\begin{abstract}
The change of impedance per unit length in a single or double conductor line situated parallel to an infinitely long two-layered metallic circular cylinder is found (within the quasistatic approximation) in the form of an infinite series. The cylinder consists of an inner core and an outer annulus. The properties of the inner core are assumed to be constant. The relative magnetic permeability, $\mu(r)=r^{\alpha}$, and the conductivity, $\sigma(r)=\sigma^{(0)} r^{\kappa}$, of the outer annulus vary with respect to the radial coordinate, $r$, and $\alpha$ and $\kappa$ are arbitrary real numbers. Numerical results are presented in the form of figures and tables.
\end{abstract}

\section{Introduction}

Eddy current methods are widely used for nondestructive testing of properties of materials, for example, in quality control of thermal processing and measurement of surface coverings. In many applications the external magnetic field modifies the properties of the material and, in particular, the magnetic permeability $\mu$ of the medium (see, for example, [2], [1]). Since almost all known analytical or closed-form solutions to eddy current testing problems deal with the cases where the properties of the material are assumed to be constant [8], there is a need to construct analytical solutions in the case where the properties of the medium vary with the spatial coordinates. For some particular cases such solutions are known (see, for example, [4]-[6] and [9]).

In practice, eddy current probes have different configurations, the most common one being the circular form. But, sometimes, it is useful to consider noncircular configurations. A long rectangular excitation frame is an example of a noncircular configuration. It is shown in [11] that a probe with a width-to-length ratio equal to 1:4 or smaller can be modelled by means of two infinitely long excitation wires instead of a rectangular wire.

\footnotetext{
${ }^{1}$ Dept of Applied Mathematics, Riga Technical University, Riga, Latvia, LV 1010

${ }^{2}$ Depts of Mathematics and Computer Science, University of Ottawa, Ottawa, ON, Canada K1N 6N5

(C) Australian Mathematical Society, 1996, Serial-fee code 0334-2700/96
} 
This paper presents a series solution for the change of impedance in a double conductor line parallel to a double-layered metallic cylinder in the case where the relative magnetic permeability, $\mu(r)$, and the conductivity, $\sigma(r)$, of the outer cylinder are functions of the radial coordinate, $r$, of the form $\mu(r)=r^{\alpha}, \sigma(r)=\sigma^{(0)} r^{k}$, where $\alpha$ and $\kappa$ are arbitrary real numbers. The solution is found, first, for the case of a single wire and then, by means of the superposition principle, for the case of two wires. Results of numerical computations are presented in the figures and tables.

\section{Governing equations}

Consider a double conductor line parallel to a two-layered metallic cylinder which consists of two concentric circular parts, an inner core and an outer annular cylinder of inner and outer radii $\tilde{\rho}_{2}$ and $\tilde{\rho}_{1}$, respectively. In Figure 1 , the two lines, $w_{1}$ and $w_{2}$, are at distances $r_{1}$ and $r_{2}$, respectively, from the cylinder axis, and are shown as lying above the cylinder in a horizontal plane perpendicular to the polar axis $\varphi=0$ (indicated as the $r$-axis in the figure).

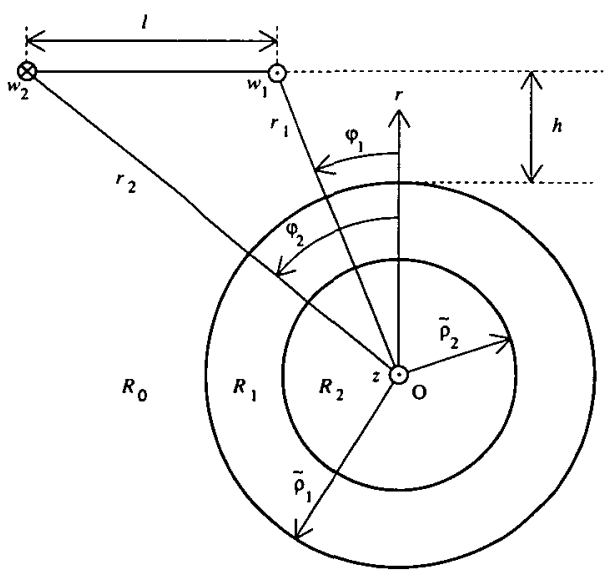

FIGURE 1. Double conductor line, in free space, lying in a horizontal plane above, and parallel to, a two-layered conducting circular cylinder.

The conductivity, $\sigma_{2}$, and the relative magnetic permeability, $\mu_{2}$, of the inner cylinder are assumed to be constant, but for the outer cylinder, these are given by

$$
\sigma_{1}(r)=\sigma^{(0)} \cdot(r / l)^{\kappa}, \quad \mu_{1}(r)=(r / l)^{\alpha},
$$

where $\alpha$ and $\kappa$ are arbitrary real numbers, $\sigma^{(0)}=$ const and $l$ is the distance between the wires. A solution to a similar problem for the case $\sigma_{1}=$ const, $\mu_{1}=$ const is given 
in [10]. We introduce a system of cylindrical polar coordinates $(r, \varphi, z)$ centered at $O$ and take the $z$-axis to be parallel to the axis of the cylinder; hence the two wires of the double conductor line are parallel to the $z$-axis.

Since relatively low frequencies are typically used in eddy current applications, we neglect the displacement current by making the quasistatic approximation in Maxwell's equations [7] which then reduce to the following three vector equations:

$$
\begin{aligned}
\operatorname{curl} \mathbf{E} & =-\frac{\partial \mathbf{B}}{\partial t}, \\
\operatorname{curl} \mathbf{H} & =\sigma(r) \mathbf{E}+\mathbf{I}^{e}, \\
\mathbf{B} & =\mu_{0} \mu(r) \mathbf{H},
\end{aligned}
$$

where $\mathbf{E}$ and $\mathbf{H}$ are the electric and magnetic field intensities, respectively, $\mathbf{B}$ is the magnetic induction vector, $\mathbf{I}^{e}$ is the external current density, $\sigma(r)$ is the conductivity of the medium and $\mu_{0}$ and $\mu(r)$ are, respectively, the magnetic constant and the relative magnetic permeability of the medium.

Introducing the vector potential $\mathbf{A}$ by the relation

$$
\mathbf{B}=\operatorname{curl} \mathbf{A}
$$

and using (2) we obtain

$$
\operatorname{curl}\left(\mathbf{E}+\frac{\partial \mathbf{A}}{\partial t}\right)=0 .
$$

Thus the vector with vanishing curl in (6) can be written as the gradient of the scalar potential $\psi$ :

$$
\mathbf{E}=-\frac{\partial \mathbf{A}}{\partial t}-\operatorname{grad} \psi
$$

Using (3)-(5) and (7) we obtain

$$
\operatorname{curl}\left(\frac{1}{\mu_{0} \mu(r)} \operatorname{curl} \mathbf{A}\right)=-\sigma(r) \frac{\partial \mathbf{A}}{\partial t}-\sigma(r) \operatorname{grad} \psi+\mathbf{I}^{e} .
$$

We assume that the functions $\mathbf{A}, \psi$ and $\mathbf{I}^{e}$ in (8) are periodic in $t$, that is

$$
\begin{aligned}
& \mathbf{A}(r, \varphi, z, t)=\widetilde{\mathbf{A}}(r, \varphi, z) e^{j \omega t}, \\
& \psi(r, \varphi, z, t)=\widetilde{\psi}(r, \varphi, z) e^{j \omega t}, \\
& \mathbf{I}^{e}(r, \varphi, z, t)=\widetilde{\mathbf{I}}^{e}(r, \varphi, z) e^{j \omega t},
\end{aligned}
$$

where $j=\sqrt{-1}$. 
Due to the geometry of the problem shown in Figure 1, we can assume that the vector potential $\tilde{\mathbf{A}}$ is parallel to the $z$-axis,

$$
\widetilde{\mathbf{A}}(r, \varphi, z)=(0,0, A(r, \varphi)),
$$

and its $z$-component, $A(r, \varphi)$, is independent of $z$.

Using (8)-(10) and basic formulae of vector calculus we obtain

$$
\begin{aligned}
& \frac{1}{\mu_{0} \mu(r)} \operatorname{grad} \operatorname{div} \widetilde{\mathbf{A}}-\frac{1}{\mu_{0} \mu(r)} \Delta \widetilde{\mathbf{A}}-\frac{1}{\mu_{0} \mu(r)^{2}} \frac{d \mu}{d r} \mathbf{e}_{r} \times \operatorname{curl} \tilde{\mathbf{A}} \\
& =-j \omega \sigma(r) \tilde{\mathbf{A}}-\sigma(r) \operatorname{grad} \tilde{\psi}+\widetilde{\mathbf{I}}^{e} .
\end{aligned}
$$

If we use the gauge

$$
\frac{1}{\mu_{0} \mu(r)} \operatorname{grad} \operatorname{div} \widetilde{\mathbf{A}}=-\sigma(r) \operatorname{grad} \tilde{\psi},
$$

the $z$-component of the vector equation (11) becomes

$$
\begin{aligned}
\frac{\partial^{2} A}{\partial r^{2}} & +\frac{1}{r} \frac{\partial A}{\partial r}+\frac{1}{r^{2}} \frac{\partial^{2} A}{\partial \varphi^{2}}-\frac{1}{\mu} \frac{d \mu}{d r} \frac{\partial A}{\partial r}-j \omega \sigma(r) \mu_{0} \mu(r) A \\
& =-\mu_{0} \mu(r) I^{e},
\end{aligned}
$$

where $I^{e}$ is the $z$-component of the vector $\widetilde{\mathbf{I}}^{e}$, and $\sigma(r)$ and $\mu(r)$ are, respectively, the conductivity and the relative magnetic permeability of the medium.

We need to solve equation (12) in the three regions $R_{0}, R_{1}$ and $R_{2}$ (see Figure 1) where $R_{0}$ is free space (containing air) described by $\tilde{\rho}_{1}<r<\infty, 0 \leq \varphi \leq 2 \pi$, $-\infty<z<\infty, R_{1}$ is the outer metallic cylindrical layer, $\tilde{\rho}_{2}<r<\widetilde{\rho}_{1}, 0 \leq \varphi \leq 2 \pi$, $-\infty<z<\infty$, with $\sigma_{1}=\sigma_{1}(r), \mu_{1}=\mu_{1}(r)$, and $R_{2}$ is the inner metallic cylinder, $0 \leq r<\widetilde{\rho}_{2}, 0 \leq \varphi \leq 2 \pi,-\infty<z<\infty$ with $\sigma_{2}=$ const and $\mu_{2}=$ const.

If we denote by $A_{i}(r, \varphi), i=0,1,2$, the vector potential in each of the three regions $R_{0}, R_{1}$ and $R_{2}$, respectively, then equation (12) splits into the three partial differential equations

$$
\begin{gathered}
\frac{\partial^{2} A_{0}}{\partial r^{2}}+\frac{1}{r} \frac{\partial A_{0}}{\partial r}+\frac{1}{r^{2}} \frac{\partial^{2} A_{0}}{\partial \varphi^{2}}= \\
+\mu_{0} I \delta\left(r-r_{1}\right) \delta\left(\varphi-\varphi_{1}\right) \\
+\mu_{0} I \delta\left(r-r_{2}\right) \delta\left(\varphi-\varphi_{2}\right), \\
\frac{\partial^{2} A_{1}}{\partial r^{2}}+\left[\frac{1}{r}-\frac{1}{\mu_{1}(r)} \frac{d \mu_{1}}{d r}\right] \frac{\partial A_{1}}{\partial r}+\frac{1}{r^{2}} \frac{\partial^{2} A_{1}}{\partial \varphi^{2}}-j \omega \sigma_{1}(r) \mu_{0} \mu_{1}(r) A_{1}=0 \\
\frac{\partial^{2} A_{2}}{\partial r^{2}}+\frac{1}{r} \frac{\partial A_{2}}{\partial r}+\frac{1}{r^{2}} \frac{\partial^{2} A_{2}}{\partial \varphi^{2}}-j \omega \sigma_{2} \mu_{0} \mu_{2} A_{2}=0
\end{gathered}
$$




\section{Mathematical analysis}

We introduce the dimensionless variable $\tilde{r}=r / l$, where the distance, $l$, between the wires is chosen as the unit of length, and recall from (1) that $\mu_{1}(\tilde{r})=\tilde{r}^{\alpha}$ and $\sigma_{1}(\tilde{r})=\sigma^{(0)} \tilde{r}^{x}$. Henceforth, tildes will be omitted. We note that now $r_{1}$ and $r_{2}$ are dimensionless parameters.

From (13)-(15), we obtain the following system of equations:

$$
\begin{aligned}
& \frac{\partial^{2} A_{0}}{\partial r^{2}}+\frac{1}{r} \frac{\partial A_{0}}{\partial r}+\frac{1}{r^{2}} \frac{\partial^{2} A_{0}}{\partial \varphi^{2}}=-\mu_{0} I l^{2} \delta\left(r-r_{1}\right) \delta\left(\varphi-\varphi_{1}\right) \\
& +\mu_{0} I l^{2} \delta\left(r-r_{2}\right) \delta\left(\varphi-\varphi_{2}\right), \\
& \frac{\partial^{2} A_{1}}{\partial r^{2}}+\frac{1-\alpha}{r} \frac{\partial A_{1}}{\partial r}+\frac{1}{r^{2}} \frac{\partial^{2} A_{1}}{\partial \varphi^{2}}-j \beta_{1}^{2} r^{\alpha+\kappa} A_{1}=0, \\
& \frac{\partial^{2} A_{2}}{\partial r^{2}}+\frac{1}{r} \frac{\partial A_{2}}{\partial r}+\frac{1}{r^{2}} \frac{\partial^{2} A_{2}}{\partial \varphi^{2}}-j \beta_{2}^{2} A_{2}=0,
\end{aligned}
$$

where

$$
\beta_{1}=l \sqrt{\omega \sigma^{(0)} \mu_{0}}, \quad \beta_{2}=l \sqrt{\omega \sigma_{2} \mu_{0} \mu_{2}} .
$$

The boundary conditions are

$$
\begin{gathered}
\left.A_{0}\right|_{r=\rho_{1}}=\left.A_{1}\right|_{r=\rho_{1}},\left.\quad \frac{\partial A_{0}}{\partial r}\right|_{r=\rho_{1}}=\left.\frac{1}{\mu_{11}} \frac{\partial A_{1}}{\partial r}\right|_{r=\rho_{1}}, \\
\left.A_{1}\right|_{r=\rho_{2}}=\left.A_{2}\right|_{r=\rho_{2}},\left.\quad \frac{1}{\mu_{12}} \frac{\partial A_{1}}{\partial r}\right|_{r=\rho_{2}}=\left.\frac{1}{\mu_{2}} \frac{\partial A_{2}}{\partial r}\right|_{r=\rho_{2}},
\end{gathered}
$$

where $\mu_{11}=\rho_{1}^{\alpha}, \mu_{12}=\rho_{2}^{\alpha}$ and $\rho_{1}=\tilde{\rho}_{1} / l, \rho_{2}=\tilde{\rho}_{2} / l$.

Since system (16)-(18) is linear, its solution can be expressed as the sum of two solutions corresponding to the presence of the wires $w_{1}$ and $w_{2}$, respectively. For the first solution, only the first term on the right-hand side of (16) is present. For the second solution, only the second term on the right-hand side of (16) is present and $r_{1}$, $\varphi_{1}$ and $I$ are replaced by $r_{2}, \varphi_{2}$ and $-I$, respectively, in the first solution. Finally, the solution to (16)-(20) is the sum of these two solutions.

To obtain the first solution, we expand Dirac's delta function in a Fourier series,

$$
\delta\left(\varphi-\varphi_{1}\right)=\frac{1}{\pi} \sum_{n=0}^{\infty} \delta_{n} \cos n\left(\varphi-\varphi_{1}\right),
$$


where

$$
\delta_{n}= \begin{cases}1 / 2, & n=0, \\ 1, & n=1,2, \ldots,\end{cases}
$$

and seek the solution to (16)-(20) in the form

$$
A_{i}(r, \varphi)=\frac{1}{\pi} \sum_{n=0}^{\infty} \delta_{n} a_{i n}(r) \cos n\left(\varphi-\varphi_{1}\right) .
$$

Substituting (21) into (16)-(20) we obtain the following system of ordinary differential equations:

$$
\begin{aligned}
\frac{d^{2} a_{0 n}}{d r^{2}}+\frac{1}{r} \frac{d a_{0 n}}{d r}-\frac{n^{2}}{r^{2}} a_{0 n} & =-\mu_{0} I l^{2} \delta\left(r-r_{1}\right), \\
\frac{d^{2} a_{1 n}}{d r^{2}}+\frac{1-\alpha}{r} \frac{d a_{1 n}}{d r}-\frac{n^{2}}{r^{2}} a_{1 n}-j \beta_{1}^{2} r^{\alpha+\kappa} a_{1 n} & =0, \\
\frac{d^{2} a_{2 n}}{d r^{2}}+\frac{1}{r} \frac{d a_{2 n}}{d r}-\frac{n^{2}}{r^{2}} a_{2 n}-j \beta_{2}^{2} a_{2 n} & =0,
\end{aligned}
$$

with the boundary conditions:

$$
\begin{aligned}
& \left.a_{0 n}\right|_{r=\rho_{1}}=\left.a_{1 n}\right|_{r=\rho_{1}},\left.\quad \frac{d a_{0 n}}{d r}\right|_{r=\rho_{1}}=\left.\frac{1}{\mu_{11}} \frac{d a_{1 n}}{d r}\right|_{r=\rho_{1}}, \\
& \left.a_{1 n}\right|_{r=\rho_{2}}=\left.a_{2 n}\right|_{r=\rho_{2}},\left.\quad \frac{1}{\mu_{12}} \frac{d a_{1 n}}{d r}\right|_{r=\rho_{2}}=\left.\frac{1}{\mu_{2}} \frac{d a_{2 n}}{d r}\right|_{r=\rho_{2}} .
\end{aligned}
$$

We begin by finding bounded general solutions to equations (22), (23) and (24).

The structure of the solution to (22) depends on the value of $n$. It will be convenient to split region $R_{0}$ into two subregions $R_{00}$ and $R_{01}$, namely,

$$
\begin{array}{ll}
R_{00}: & \rho_{1}<r<r_{1}, \quad 0 \leq \varphi \leq 2 \pi, \quad-\infty<z<\infty, \text { and } \\
R_{01}: & r>r_{1}, \quad 0 \leq \varphi \leq 2 \pi, \quad-\infty<z<\infty .
\end{array}
$$

First, if $n=0$, we denote the general solution of (22) in $R_{00}$ and $R_{01}$ by $a_{00}^{(0)}(r)$ and $a_{00}^{(1)}(r)$, respectively. Bounded general solutions to (22) in these two regions can be written, respectively, in the form

$$
\begin{aligned}
& a_{00}^{(0)}(r)=C_{10} \ln r+C_{20}, \\
& a_{00}^{(1)}(r)=C_{30} .
\end{aligned}
$$

Second, if $n \neq 0$, bounded general solutions to (22) in $R_{00}$ and $R_{01}$ are, respectively,

$$
\begin{aligned}
& a_{0 n}^{(0)}(r)=C_{1 n} r^{n}+C_{2 n} r^{-n}, \\
& a_{0 n}^{(1)}(r)=C_{3 n} r^{-n} .
\end{aligned}
$$


The general solution to equation (23) can be expressed in terms of Bessel functions (see [3]):

$$
a_{1 n}(r)=C_{4 n} r^{a} J_{p}\left(b r^{c}\right)+C_{5 n} r^{a} Y_{p}\left(b r^{c}\right)
$$

where

$a=\frac{\alpha}{2}, \quad c=1+(\alpha+\kappa) / 2, \quad b=\beta_{1} \sqrt{-j} / c, \quad p=\sqrt{\alpha^{2}+4 n^{2}} /(\kappa+\alpha+2)$.

A bounded general solution to (24) has the form

$$
a_{2}(r)=C_{6 n} J_{n}(k r)
$$

where $k=\beta_{2} \sqrt{-j}$.

Note that the structure of (31) and (32) remains the same for $n=0,1,2, \ldots$, except for the case $\alpha+\kappa=-2$, where equation (23) degenerates into Euler's equation.

We now use the boundary conditions (25) and (26) to determine the arbitrary constants in the general solutions (27)-(32).

First, we consider the case $n=0$. Since the vector potential is continuous at $r=r_{1}$ we have

$$
a_{00}^{(0)}\left(r_{1}\right)=a_{00}^{(1)}\left(r_{1}\right), \quad \text { or } \quad C_{10} \ln r_{1}+C_{20}=C_{30} .
$$

Multiplying (22) by $r$, integrating with respect to $r$ from $r_{1}-\varepsilon$ to $r_{1}+\varepsilon$ and taking the limit as $\varepsilon \rightarrow+0$, we obtain

$$
\left.\frac{d a_{02}}{d r}\right|_{r=r_{1}}-\left.\frac{d a_{01}}{d r}\right|_{r=r_{1}}=-\mu_{0} I l^{2}
$$

Using (25), (26), (33) and (34) to determine the constants $C_{10}, C_{20}, \ldots, C_{60}$ in (27), (28), (31) and (32) for $n=0$, we obtain

$$
\begin{aligned}
& C_{10}=\mu_{0} I l^{2} r_{1}, \\
& C_{20}=\rho_{1}^{a}\left[d_{0} J_{p}\left(b \rho_{1}^{c}\right)+Y_{p}\left(b \rho_{1}^{c}\right)\right] C_{50}-\mu_{0} I l^{2} r_{1} \ln \rho_{1}, \\
& C_{30}=C_{20}+C_{10} \ln r_{1}, \\
& C_{40}=d_{0} C_{50}, \\
& C_{50}=\frac{\mu_{0} \mu_{11} I l^{2} r_{1} \rho_{2}^{-a}}{d_{0}\left[a J_{p}\left(b \rho_{1}^{c}\right)+b c \rho_{1}^{c} J_{p}^{\prime}\left(b \rho_{1}^{c}\right)\right]+a Y_{p}\left(b \rho_{1}^{c}\right)+b c \rho_{1}^{c} Y_{p}^{\prime}\left(b \rho_{1}^{c}\right)}, \\
& C_{60}=\frac{\rho_{2}^{\alpha}\left[C_{40} J_{p}\left(b \rho_{2}^{c}\right)+C_{50} Y_{p}\left(b \rho_{2}^{c}\right)\right]}{J_{0}\left(k \rho_{2}\right)},
\end{aligned}
$$


where

$$
d_{0}=-\frac{\mu_{12} k \rho_{2} J_{0}^{\prime}\left(k \rho_{2}\right) Y_{p}\left(b \rho_{2}^{c}\right)-\mu_{2} J_{0}\left(k \rho_{2}\right)\left[a Y_{p}\left(b \rho_{2}^{c}\right)+b c \rho_{2}^{c} Y_{p}^{\prime}\left(b \rho_{2}^{c}\right)\right]}{\mu_{12} k \rho_{2} J_{0}^{\prime}\left(k \rho_{2}\right) J_{p}\left(b \rho_{2}^{c}\right)-\mu_{2} J_{0}\left(k \rho_{2}\right)\left[a J_{p}\left(b \rho_{2}^{c}\right)+b c \rho_{2}^{c} J_{p}^{\prime}\left(b \rho_{2}^{c}\right)\right]}
$$

Since we are interested in the change of impedance in the double conductor line due to the presence of the conducting cylinder, by (10) we need only determine the $z$-component, $A_{0}(r, \varphi)$, of the vector potential in $R_{0}$.

In free space $R_{0}, A_{0}(r, \varphi)$ can be written as the sum of two terms,

$$
A_{0}(r, \varphi)=A_{0}^{\text {empty }}(r, \varphi)+A_{0}^{i n d}(r, \varphi)
$$

where the first term is the vector potential due to the double conductor line in empty unbounded free space and the second term is the induced vector potential due to the conducting cylinder. We shall be concerned only with the induced term because it is the important term in eddy current testing.

Again, in $R_{0}$, the induced potential $A_{0}^{i n d}(r, \varphi)$ can be written as the sum of two parts:

$$
A_{0}^{i n d}(r, \varphi)=A_{0}^{\left(w_{1}\right)}(r, \varphi)+A_{0}^{\left(w_{2}\right)}(r, \varphi),
$$

where the first and second terms on the right-hand side correspond to the wires $w_{1}$ and $w_{2}$, respectively. In Figure 1, the cylindrical polar coordinates of the $i$ th wire are $r=r_{i}, \varphi=\varphi_{i},-\infty<z<\infty$, for $i=1,2$.

The first term of the Fourier series of $A_{0}^{\left(w_{1}\right)}(r, \varphi)$ is equal to $C_{20}$ and can be written in the form

$$
a_{0}^{\left(w_{1}\right)}(r)=\frac{\mu_{0} \mu_{11} I l^{2} r_{1} \rho_{2}^{-a} \rho_{1}^{a}\left[d_{0} J_{p}\left(b \rho_{1}^{c}\right)+Y_{p}\left(b \rho_{1}^{c}\right)\right]}{d_{0}\left[a J_{p}\left(b \rho_{1}^{c}\right)+b c \rho_{1}^{c} J_{p}^{\prime}\left(b \rho_{1}^{c}\right)\right]+a Y_{p}\left(b \rho_{1}^{c}\right)+b c \rho_{1}^{c} Y_{p}^{\prime}\left(b \rho_{1}^{c}\right)}
$$

Second, we determine the arbitrary constants in the cases $n=1,2, \ldots$ As in the case $n=0$, we obtain additional conditions at $r=r_{1}$ in the form

$$
\begin{gathered}
\left.a_{2 n}\right|_{r=r_{1}}=\left.a_{1 n}\right|_{r=r_{1}}, \\
\left.\frac{d a_{2 n}}{d r}\right|_{r=r_{1}}-\left.\frac{d a_{1 n}}{d r}\right|_{r=r_{1}}=-\mu_{0} I l^{2} .
\end{gathered}
$$

Using (25), (26), (35) and (36) to determine the constants $C_{1 n}, C_{2 n}, \ldots, C_{6 n}$ in 
(29)-(32), we obtain

$$
\begin{aligned}
& C_{1 n}=\frac{\mu_{0}}{2 n} I l^{2} r_{1}^{-n+1}, \\
& C_{2 n}=a_{11} / a_{12}, \\
& C_{3 n}=C_{2 n}+C_{1 n} r_{1}^{2 n}, \\
& C_{4 n}=d_{n} C_{5 n}, \\
& C_{5 n}=\frac{2 n \rho_{1}^{-2 n} C_{2 n}+\mu_{0} I l^{2} r_{1}^{-n+1}}{2 n \rho_{1}^{a-n}\left[d_{n} J_{p}\left(b \rho_{1}^{c}\right)+Y_{p}\left(b \rho_{1}^{c}\right)\right]}, \\
& C_{6 n}=\frac{\rho_{2}^{a}\left[C_{4 n} J_{p}\left(b \rho_{2}^{c}\right)+C_{5 n} Y_{p}\left(b \rho_{2}^{c}\right)\right]}{J_{n}\left(k \rho_{2}\right)},
\end{aligned}
$$

where

$$
\begin{aligned}
d_{n}=- & \frac{\mu_{12} k \rho_{2} J_{n}^{\prime}\left(k \rho_{2}\right) Y_{p}\left(b \rho_{2}^{c}\right)-\mu_{2} J_{n}\left(k \rho_{2}\right)\left[a Y_{p}\left(b \rho_{2}^{c}\right)+b c \rho_{2}^{c} Y_{p}^{\prime}\left(b \rho_{2}^{c}\right)\right]}{\mu_{12} k \rho_{2} J_{n}^{\prime}\left(k \rho_{2}\right) J_{p}\left(b \rho_{2}^{c}\right)-\mu_{2} J_{n}\left(k \rho_{2}\right)\left[a J_{p}\left(b \rho_{2}^{c}\right)+b c \rho_{2}^{c} J_{p}^{\prime}\left(b \rho_{2}^{c}\right)\right]} \\
a_{11}= & \frac{\mu_{0} I l^{2} r_{1}^{-n+1} \rho_{1}^{2 n}}{2 n}\left\{\mu_{11} n\left[d_{n} J_{p}\left(b \rho_{1}^{c}\right)+Y_{p}\left(b \rho_{1}^{c}\right)\right]\right. \\
& \left.-d_{n}\left[a J_{p}\left(b \rho_{1}^{c}\right)+b c \rho_{1}^{c} J_{p}^{\prime}\left(b \rho_{1}^{c}\right)\right]-a Y_{p}\left(b \rho_{1}^{c}\right)-b c \rho_{1}^{c} Y_{p}\left(b \rho_{1}^{c}\right)\right\} \\
a_{12}= & \mu_{11} n\left[d_{n} J_{p}\left(b \rho_{1}^{c}\right)+Y_{p}\left(b \rho_{1}^{c}\right)\right]+d_{n}\left[a J_{p}\left(b \rho_{1}^{c}\right)\right. \\
& \left.+b c \rho_{1}^{c} J_{p}^{\prime}\left(b \rho_{1}^{c}\right)\right]+a Y_{p}\left(b \rho_{1}^{c}\right)+b c \rho_{1}^{c} Y_{p}^{\prime}\left(b \rho_{1}^{c}\right)
\end{aligned}
$$

Thus, with $q=\alpha /(\kappa+\alpha+2)$, we have

$$
\begin{aligned}
A_{0}^{\left(w_{1}\right)}(r, \varphi)= & \frac{\mu_{0} I l^{2} r_{1}}{2 \pi}\left\{\frac{\mu_{11} \rho_{2}^{-a} \rho_{1}^{a}\left[d_{0} J_{q}\left(b \rho_{1}^{c}\right)+Y_{q}\left(b \rho_{1}^{c}\right)\right]}{d_{0}\left[a J_{q}\left(b \rho_{1}^{c}\right)+b c \rho_{1}^{c} J_{q}^{\prime}\left(b \rho_{1}^{c}\right)\right]+a Y_{q}\left(b \rho_{1}^{c}\right)+b c \rho_{1}^{c} Y_{q}^{\prime}\left(b \rho_{1}^{c}\right)}\right. \\
& \left.+\sum_{n=1}^{\infty} \frac{\rho_{1}^{2 n}}{n r_{1}^{n} r^{n}} \frac{b_{11}}{b_{12}} \cos n\left(\varphi-\varphi_{1}\right)\right\}
\end{aligned}
$$

where

$$
\begin{aligned}
b_{11}= & \mu_{11} n\left[d_{n} J_{p}\left(b \rho_{1}^{c}\right)+Y_{p}\left(b \rho_{1}^{c}\right)\right]-d_{n}\left[a J_{p}\left(b \rho_{1}^{c}\right)+b c \rho_{1}^{c} J_{p}^{\prime}\left(b \rho_{1}^{c}\right)\right] \\
& -a Y_{p}\left(b \rho_{1}^{c}\right)-b c \rho_{1}^{c} Y_{p}^{\prime}\left(b \rho_{1}^{c}\right), \\
b_{12}= & \mu_{11} n\left[d_{n} J_{p}\left(b \rho_{1}^{c}\right)+Y_{p}\left(b \rho_{1}^{c}\right)\right]+d_{n}\left[a J_{p}\left(b \rho_{1}^{c}\right)+b c \rho_{1}^{c} J_{p}^{\prime}\left(b \rho_{1}^{c}\right)\right] \\
& +a Y_{p}\left(b \rho_{1}^{c}\right)+b c \rho_{1}^{c} Y_{p}^{\prime}\left(b \rho_{1}^{c}\right)
\end{aligned}
$$

and $d_{n}$ is defined by (37). 
To obtain the second part, $A_{0}^{\left(w_{2}\right)}(r, \varphi)$, of the induced vector potential which corresponds to the second term on the right-hand side of (16), in (38) we replace $r_{1}, \varphi_{1}$ and $I$ with $r_{2}, \varphi_{2}$ and $-I$, respectively.

Adding $A_{0}^{\left(w_{1}\right)}(r, \varphi)$ and $A_{0}^{\left(w_{2}\right)}(r, \varphi)$, we obtain the induced vector potential in the form

$$
\begin{aligned}
A_{0}^{i n d}(r, \varphi)= & \frac{\mu_{0} I l^{2}\left(r_{1}-r_{2}\right)}{2 \pi} \\
& \times \frac{\mu_{11} \rho_{2}^{-a} \rho_{1}^{a}\left[d_{0} J_{p}\left(b \rho_{1}^{c}\right)+Y_{p}\left(b \rho_{1}^{c}\right)\right]}{d_{0}\left[a J_{p}\left(b \rho_{1}^{c}\right)+b c \rho_{1}^{c} J_{p}^{\prime}\left(b \rho_{1}^{c}\right)\right]+a Y_{p}\left(b \rho_{1}^{c}\right)+b c \rho_{1}^{c} Y_{p}^{\prime}\left(b \rho_{1}^{c}\right)} \\
& +\frac{\mu_{0} I l^{2}}{2 \pi}\left[r_{1} \sum_{n=1}^{\infty} \frac{\rho_{1}^{2 n}}{n r_{1}^{n} r^{n}} \frac{b_{11}}{b_{12}} \cos n\left(\varphi-\varphi_{1}\right)\right. \\
& \left.-r_{2} \sum_{n=1}^{\infty} \frac{\rho_{1}^{2 n}}{n r_{2}^{n} r^{n}} \frac{b_{11}}{b_{12}} \cos n\left(\varphi-\varphi_{2}\right)\right] .
\end{aligned}
$$

The change of impedance in the double conductor line due to the conducting cylinder can be found by the formula

$$
Z^{i n d}=\frac{j \omega}{I} \oint_{L} A_{0}^{i n d}(r, \varphi) d l,
$$

where $L$ is the contour of integration. In our model we assume that integration takes place along the two wires in the opposite directions over one unit of length. Hence, from (39) and (40) we obtain

$$
Z^{i n d}=\frac{\omega \mu_{0} l^{2}}{2 \pi} Z_{0}
$$

where

$$
\begin{aligned}
Z_{0}= & j \sum_{n=1}^{\infty} \frac{b_{11}}{n b_{12}}\left[r_{1}\left(\frac{\rho_{1}}{r_{1}}\right)^{2 n}+r_{2}\left(\frac{\rho_{1}}{r_{2}}\right)^{2 n}\right. \\
& \left.-\left(r_{1}+r_{2}\right)\left(\frac{\rho_{1}}{r_{1}}\right)^{n}\left(\frac{\rho_{1}}{r_{2}}\right)^{n} \cos n\left(\varphi_{1}-\varphi_{2}\right)\right] .
\end{aligned}
$$

\section{Numerical results}

Formula (41) was used to compute the change of impedance in the double conductor line for different values of the parameters of the problem. The series in (41) was computed by means of MATHEMATICA (version 2.2.2) on a Sun Sparc 10, because 
this package easily allows the evaluation of Bessel functions of fractional order for a complex argument. The graphs in Figures $2-4$ correspond to the case where

$$
\begin{aligned}
& \mu_{1}(r)=r^{\alpha}, \quad \sigma_{1}(r)=\sigma^{(0)}=\text { const } \quad(\kappa=0), \\
& \mu_{11}=\rho_{1}^{\alpha}, \quad \mu_{12}=\rho_{2}^{\alpha}, \quad \mu_{2}=1, \quad \beta_{2}=1 .
\end{aligned}
$$

In Figures 2-5, the variable $x=r_{1} \sin \varphi_{1}+l / 2$ describes the horizontal shift of the centre of the double conductor line measured from the vertical axis $\varphi=0$ (denoted as the $r$-axis in Figure 1).

In Figure 2, the modulus $\left|Z_{0}\right|$ of $Z_{0}$ is plotted against $x$ for different values of the parameter $\beta_{1}$. The remaining paramaters are set at

$$
\rho_{1}=1.1, \quad \rho_{2}=1.0, \quad h=0.4, \quad \alpha=-1 .
$$

As can be seen, $\left|Z_{0}\right|$ increases with $\beta_{1}$.

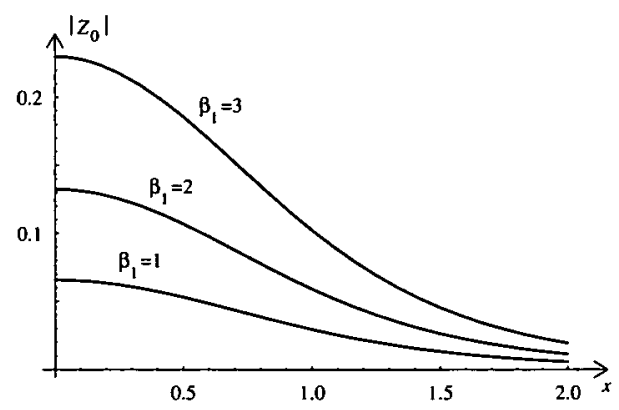

FIGURE 2. $\left|Z_{0}\right|$ against $x$ for $\beta_{1}=1,2,3$.

In Figure 3, $\left|Z_{0}\right|$ is plotted against $x$ for $\alpha=-1,-3 / 2,-3$, respectively. The values of the remaining parameters are

$$
\rho_{1}=0.4, \quad \rho_{2}=0.3, \quad \beta_{1}=1, \quad \beta_{2}=1, \quad h=0.1 .
$$

It is seen that $\left|Z_{0}\right|$ increases as the change of magnetic permeability across the layer $\rho_{2}<r<\rho_{1}$ becomes stronger, a fact which is important in qualitative analysis of eddy current testing of media with varying properties.

Another interesting phenomenon can be seen in Figure 4, where $\left|Z_{0}\right|$ is plotted against $x$ for different values of $\rho_{2}$ and $\rho_{1}$.

If the radii of the cylinders are small in comparison with the distance between the wires of the double conductor line, then the local minimum of $\left|Z_{0}\right|$ (as a function of $x$ ) is shifted away from the origin (see Figures 4-5). This may be useful for quantitative 


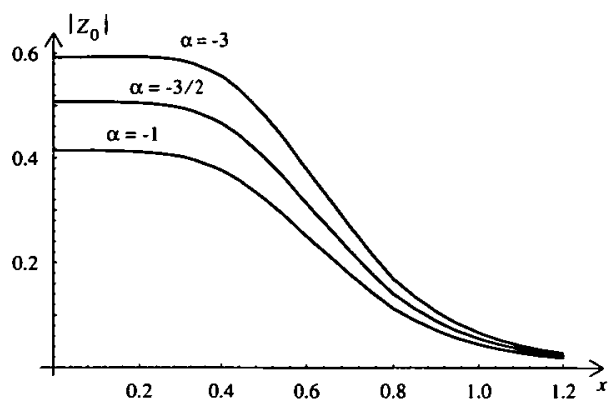

FIGURE 3. $\left|Z_{0}\right|$ against $x$ for magnetic permeability $\mu_{1}(r)=r^{\alpha}$ of medium $R_{1}$, where $\alpha=$ $-1,-3 / 2,-3$.

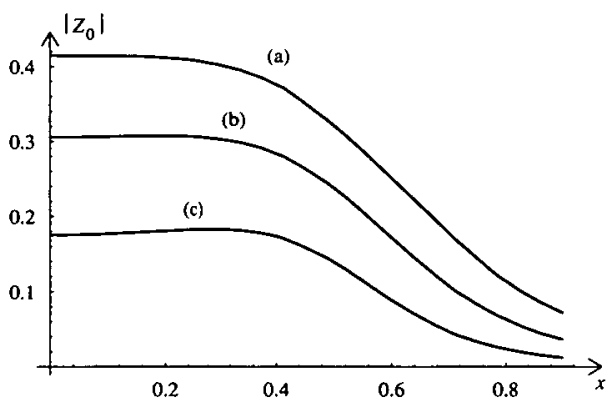

FIGURE 4. $\left|Z_{0}\right|$ against $x$ for $h=0.1$ and the following values of $\rho_{1}$ and $\rho_{2}$ : (a) $\rho_{1}=0.4, \rho_{2}=0.3$, (b) $\rho_{1}=0.3, \rho_{2}=0.2$, (c) $\rho_{\mathrm{t}}=0.2, \rho_{2}=0.1$.

control of materials with varying properties. The other parameters in Figure 4 are set at

$$
\alpha=-1, \quad \beta_{1}=1, \quad \beta_{2}=1 .
$$

In Figure $5,\left|Z_{0}\right|$ is plotted against $x$ for the case

$$
\mu_{1}(r)=r^{-2}, \quad \rho_{1}=0.3, \quad \rho_{2}=0.2, \quad h=0.1, \quad \beta_{1}=\beta_{2}=1 .
$$

The conductivity, $\sigma_{1}(r)$, of $R_{1}$ is of the form $\sigma_{1}(r)=\sigma^{(0)} r^{\kappa}$ for $\kappa=-1,-2,-3$.

Comparing Figures 3 and 5, one sees that the values of $\alpha$ and $\kappa$ have an opposite effect on the value of $\left|Z_{0}\right|$. It is seen from Figure 3 that $\left|Z_{0}\right|$ increases if the magnetic permeability is decreasing more rapidly in the radial direction (that is, if $\alpha$ decreases from -1 to -3 ). On the other hand, Figure 5 shows that $\left|Z_{0}\right|$ decreases if $\kappa$ decreases from -1 to -3 (that is, the conductivity, $\sigma(r)$, is decreasing more rapidly in the radial direction). 


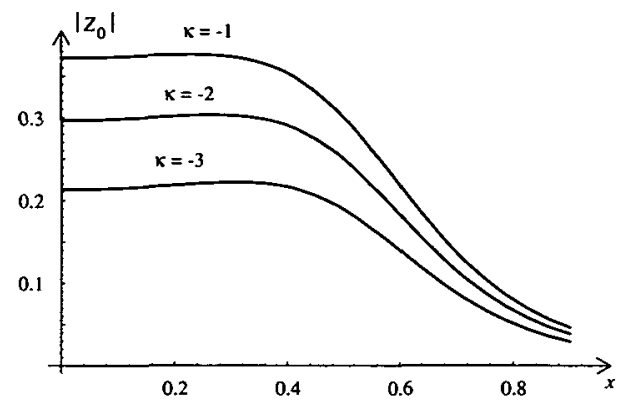

FIGURE 5. $\left|Z_{0}\right|$ against $x$ for conductivity $\sigma_{1}(r)=\sigma^{(0)} r^{k}$ of medium $R_{1}$, where $\kappa=-1,-2,-3$.

Since $\left|Z_{0}\right|$ changes slowly in a neighbourhood of $x=0$ we present two tables of data, rounded to four decimal places, corresponding to Figures 4-5.

\begin{tabular}{|c|c|c|c|}
\hline$x$ & \multicolumn{3}{|c|}{$\left|Z_{0}\right|$} \\
\hline & $\rho_{1}=0.4, \rho_{2}=0.3$ & $\rho_{1}=0.3, \rho_{2}=0.2$ & $\rho_{1}=0.2, \rho_{2}=0.1$ \\
\hline 0.0 & 0.4149 & 0.3059 & 0.1756 \\
0.1 & 0.4145 & 0.3066 & 0.1771 \\
0.2 & 0.4119 & 0.3074 & 0.1808 \\
0.3 & 0.4023 & 0.3032 & 0.1828 \\
0.4 & 0.3766 & 0.2843 & 0.1737 \\
0.5 & 0.3253 & 0.2390 & 0.1402 \\
0.6 & 0.2511 & 0.1718 & 0.0885 \\
0.7 & 0.1743 & 0.1077 & 0.0467 \\
0.8 & 0.1131 & 0.0630 & 0.0236 \\
0.9 & 0.0714 & 0.0365 & 0.0122 \\
\hline
\end{tabular}

TABLE 1. $\left|Z_{0}\right|$ against $x$ for shown values of radii, $\rho_{1}$ and $\rho_{2}$, of cylinders, and $h=0.1$.

\section{Conclusion}

The change of impedance, per unit length, in a double conductor line due to the presence of an infinitely long two-layered conducting circular cylinder which is situated parallel to the conductor line is found in the case the magnetic permeability, $\mu(r)=r^{\alpha}$, and the conductivity, $\sigma(r)=\sigma^{(0)} r^{k}$, of the outer cylindrical layer are not constant, where $\sigma^{(0)}=$ const, and $\alpha$ and $\kappa$ are arbitrary real numbers. This problem 


\begin{tabular}{|c|c|c|c|}
\hline$x$ & \multicolumn{3}{|c|}{$\left|Z_{0}\right|$} \\
\hline & $\kappa=-1$ & $\kappa=-2$ & $\kappa=-3$ \\
\hline 0.0 & 0.3722 & 0.2965 & 0.2130 \\
0.1 & 0.3735 & 0.2981 & 0.2146 \\
0.2 & 0.3760 & 0.3016 & 0.2184 \\
0.3 & 0.3738 & 0.3027 & 0.2217 \\
0.4 & 0.3544 & 0.2908 & 0.2166 \\
0.5 & 0.3012 & 0.2505 & 0.1897 \\
0.6 & 0.2177 & 0.1825 & 0.1395 \\
0.7 & 0.1365 & 0.1145 & 0.0876 \\
0.8 & 0.0797 & 0.0668 & 0.0509 \\
0.9 & 0.0461 & 0.0385 & 0.0292 \\
\hline
\end{tabular}

TABLE 2. $\left|Z_{0}\right|$ against $x$ for conductivity $\sigma_{1}(r)=\sigma^{(0)} r^{\kappa}$ of medium $R_{1}$, where $\kappa=-1,-2,-3$.

is solved by means of an infinite series containing Bessel functions. Computational results are presented in the form of figures.

\section{Acknowledgments}

This work was supported in part by the Natural Sciences and Engineering Research Council of Canada under grants OGP0007916, EQP0122258 and EQP0139636, and the Centre de recherches mathématiques of the Université de Montréal.

\section{References}

[1] N. P. Benklevskaya, V. E. Scherbinin, G. A. Burtsev and Yu. V. Stepin, "Operation on ferromagnetic probe transducers in a strongly nonuniform magnetic field", Soviet $J$. of Nondestructive Testing 24(5) (1988) 333-337.

[2] V. V. Dyakin and V. A. Sandovsky, "Theory and computations of superposed transducers" (Nauka, Moscow, 1981) (In Russian).

[3] E. Jahnke and F. Emde, Tables of Functions with Formulae and Curves (Dover, New York, 1945) 146.

[4] G. A. Kasimov and Yu. V. Kulaev, "An applied electromagnetic transducer above the object of inspection with the electrical and magnetic properties of the material changing with depth", Soviet J. of Nondestructive Testing 14 (1978) 550-552.

[5] A. A. Kolyshkin, A. P. Smolyakov, and R. Vaillancourt, "Analytical solution in eddy-current testing of double-layer media with depth-varying magnetic properties", IEEE Trans. on Magnetics 27 (1991) 4360-4365. 
[6] A. A. Kolyshkin and R. Vaillancourt, "Analytical solution to eddy current testing of cylindrical problems with varying properties", Canad. Appl. Math. Quart. 2 (Summer 1994) 349-360.

[7] W. R. Smythe, Static and Dynamic Electricity, 3rd ed. (Hemisphere, New York, 1989) 368.

[8] J. A. Tegopoulos and E. E. Kriezis, Eddy currents in linear conducting media, Studies in Electrical and Electronic Engineering, vol. 16, (Elsevier, Amsterdam, 1985).

[9] E. Uzal, J. C. Moulder, S. Mitra, and J. H. Rose, "Impedance of coils over layered metals with continuously variable conductivity and permeability: Theory and experiment", J. Appl. Phys. 74 (1993) 2076-2089.

[10] V. V. Vlasov and V. A. Komarov, "Interaction of the magnetic field of a long single-turn loop with a conducting ferromagnetic cylinder", Soviet J. of Nondestructive Testing 8 (1972) 433-440.

[11] N. N. Zatsepin and V. M. Tatamikov, "Theory of a superposed transducer", Soviet J. of Nondestructive Testing 18 (1982) 81-87. 